\title{
String production in the Abelian Higgs vacuum
}

\author{
Ayush Saurabh and Tanmay Vachaspati \\ Physics Department, Arizona State University, Tempe, Arizona 85287, USA
}

(Received 7 January 2019; published 7 May 2019)

\begin{abstract}
We numerically study string production by evolving classical Abelian Higgs gauge field wave packets. Initial conditions are constructed for the propagation of a single wave packet and for the collision of two wave packets. We identify regions of parameter space that lead to prompt production of strings from a single wave packet. The collision of two subcritical wave packets can also lead to the production of strings in certain regions of parameter space.
\end{abstract}

DOI: 10.1103/PhysRevD.99.103509

\section{INTRODUCTION}

Topological defects such as kinks, strings, and magnetic monopoles are classical solutions in a wide range of field theories. In quantum theory, topological defects can be viewed as a bound state of a large number of quanta. The interpretation of solitons as particles is most explicitly known in the sine-Gordon model. In that case, the operators that create and destroy solitons (which are fermions) can be written in terms of particle quanta that are bosons. The question of interest in this paper is if it is possible to assemble particles to make strings. And if so, can we say something about the initial conditions necessary to produce strings?

The transition from particles to solitons is difficult to treat because particles are described by quantum field theory whereas solitons are described by classical field theory. However, from a practical standpoint, we often produce high occupation number states of quantum particles that behave quasiclassically. For example, by sending currents into a light bulb we produce light that can be described as classical radiation using Maxwell's equations. Thus, it is relevant to consider the production of solitons in the scattering of classical waves or wave packets. We will restrict our attention to this situation and ask what classical initial conditions lead to the production of solitons in the final stage.

These questions were addressed in Ref. [1] for an $\mathrm{SO}(3)$ field theory, where incoming wave packets led to the production of magnetic monopoles. While the possible production of magnetic monopoles is exciting, it is in the realm of speculative physics because we do not know if

Published by the American Physical Society under the terms of the Creative Commons Attribution 4.0 International license. Further distribution of this work must maintain attribution to the author(s) and the published article's title, journal citation, and DOI. Funded by SCOAP ${ }^{3}$. grand unified theories are correct. On the other hand, strings are closer to reality since we do have superconductors in which (gauge) strings exist. In this paper we focus on the production of gauge U(1) strings, where the class of initial conditions we use are motivated by the initial conditions of Ref. [1].

There are several aspects of the string creation problem that differ from the monopole creation problem. In the latter, once monopole-antimonopole pairs are created with enough energy, they fly apart and survive indefinitely. On the other hand, only closed loops of string can be created. These oscillate, radiate, collapse, and survive only for a finite amount of time. If some of the loops are produced with large angular momentum, they live for longer but eventually decay. A second difference is that the properties of the string network that is produced change with time because the strings interact with each other and intercommute to form smaller loops.

We introduce the field theory and string solution in Sec. II followed by our choice of initial conditions in Sec. III. The computational methods used in our analysis are described in Sec. IV and then we present our results in Sec. V. We conclude in Sec. VI.

\section{ABELIAN HIGGS MODEL AND STRINGS}

We consider the Abelian Higgs model given by the Lagrangian,

$$
\mathcal{L}=-\frac{1}{4} F_{\mu \nu} F^{\mu \nu}+\frac{1}{2}\left|D_{\mu} \phi\right|^{2}-\frac{\lambda}{4}\left(|\phi|^{2}-\eta^{2}\right)^{2}
$$

where $\phi=\phi_{1}+i \phi_{2}$ is a complex scalar field, $D_{\mu}=$ $\partial_{\mu}+i e A_{\mu}, A_{\mu}$ is the gauge field with field strength tensor $F_{\mu \nu}=\partial_{\mu} A_{\nu}-\partial_{\nu} A_{\mu}$, and $\lambda$ and $e$ are coupling constants. The energy density in the fields is 
$E=\frac{1}{2}\left|D_{0} \phi\right|^{2}+\frac{1}{2}\left|D_{i} \phi\right|^{2}+\frac{1}{2}\left(\mathbf{E}^{2}+\mathbf{B}^{2}\right)+\frac{\lambda}{4}\left(|\phi|^{2}-\eta^{2}\right)^{2}$

where $E_{i}=F_{0 i}$ is the electric field and $B_{i}=\epsilon_{i j k} F_{j k} / 2$ is the magnetic field.

Topological string solutions in the Abelian Higgs model are well known. The solution for a straight string along the $z$ axis is

$$
\phi=\eta f(r) e^{i \theta}, \quad A_{i}=v(r) \epsilon_{i j} \frac{x^{j}}{r^{2}}(i, j=1,2)
$$

where we work in cylindrical coordinates $r=\sqrt{x^{2}+y^{2}}$, $\theta=\tan ^{-1}(y / x)$, and $f(r)$ and $v(r)$ are profile functions that vanish at the origin and go to 1 asymptotically. The energy per unit length (also the tension) of the string is given by

$$
\mu=\pi \eta^{2} F(\beta)
$$

where $\beta \equiv 2 \lambda / e^{2}$. The function $F(\beta)$ is known numerically and is a smooth, slowly varying function. We also have $F(1)=1$ in the so-called Bogolmol'nyi-PrasadSommerfield limit when the scalar mass in the model, $m_{S}=\sqrt{2 \lambda} \eta$ equals the vector mass, $m_{V}=e \eta$. For $\beta$ not too large, the thickness of the scalar fields in the string is $\sim m_{S}^{-1}$ and of the vector fields is $\sim m_{V}^{-1}$.

The string is characterized by a topological winding number that is defined by

$$
n=\frac{-i}{2 \pi \eta^{2}} \oint d x^{i} \phi^{*} \partial_{i} \phi=\frac{1}{2 \pi} \oint \frac{d \theta}{d l} d l
$$

where $\theta$ is the phase of the scalar field at a given point on the contour and $l$ denotes the parameter along the integration curve.

\section{INITIAL CONDITIONS}

We base the initial conditions for our simulations on those used for monopole-antimonopole production [1]. We adopt the temporal gauge for all our simulations, that is, $A_{0}=0$, and construct circularly polarized gauge wave packet configurations (not solutions) that propagate along the $\pm z$ axis. Consider the ansatz below for a wave packet propagating in the $-z$ direction,

$$
\begin{gathered}
\mathcal{A}_{x}=\partial_{y} f_{1}\left(\omega f_{2}-\partial_{z} f_{2}\right) \cos \left(\omega\left(t+z-z_{0}\right)\right) \\
\mathcal{A}_{y}=\partial_{x} f_{1}\left(\omega f_{2}+\partial_{z} f_{2}\right) \sin \left(\omega\left(t+z-z_{0}\right)\right) \\
\mathcal{A}_{z}=\partial_{x} \partial_{y} f_{1} f_{2}\left[\cos \left(\omega\left(t+z-z_{0}\right)\right)-\sin \left(\omega\left(t+z-z_{0}\right)\right)\right]
\end{gathered}
$$

where $f_{1}=f_{1}(x, y), f_{2}=f_{2}\left(t+z-z_{0}\right)$ will be specified below, and $z_{0}$ determines the initial $(t=0)$ location of the wave packet along the $z$ axis. Now the initial conditions for the gauge fields and their time derivatives are

$$
\begin{gathered}
A_{i}(t=0, \mathbf{x})=\mathcal{A}_{i}(t=0, \mathbf{x}), \\
\partial_{t} A_{i}(t=0, \mathbf{x})=\left[\partial_{t} \mathcal{A}_{i}(t, \mathbf{x})\right]_{t=0} .
\end{gathered}
$$

This form for the gauge fields satisfies $\nabla \cdot \boldsymbol{A}=0$ which will be useful later when we discuss Gauss constraints.

We can also construct a wave packet traveling in the $+z$ direction in a similar manner. To do this, we write the formulas in terms of $f_{3}\left(t-\left(z+z_{0}\right)\right)$ :

$$
\begin{aligned}
& \mathcal{A}_{x}^{\prime}=\partial_{y} f_{1}\left(-\omega^{\prime} f_{3}-\partial_{z} f_{3}\right) \cos \left(\omega^{\prime}\left(t-z-z_{0}\right)\right. \\
& \mathcal{A}_{y}^{\prime}=-\partial_{x} f_{1}\left(\omega^{\prime} f_{3}-\partial_{z} f_{3}\right) \sin \left(\omega^{\prime}\left(t-z-z_{0}\right)\right)
\end{aligned}
$$

$\mathcal{A}_{z}^{\prime}=\partial_{x} \partial_{y} f_{1} f_{3}\left(\cos \left(\omega^{\prime}\left(t-z-z_{0}\right)\right)-\sin \left(\omega^{\prime}\left(t-z-z_{0}\right)\right)\right.$.

And these can be used to construct initial conditions for a wave packet that propagates in the $+z$ direction as above.

We choose profile functions in a manner that localizes the gauge wave packet in all directions;

$$
\begin{gathered}
f_{1}(x, y)=a \exp \left[-\frac{x^{2}+y^{2}}{2 w^{2}}\right] \\
f_{2}\left(t+z-z_{0}\right)=\exp \left[-\frac{\left(t+z-z_{0}\right)^{2}}{2 w^{2}}\right] \\
\left.f_{3}\left(t-z-z_{0}\right)\right)=\exp \left[-\frac{\left(t-z-z_{0}\right)^{2}}{2 w^{2}}\right]
\end{gathered}
$$

where $a$ is the amplitude and $w$ is the width of the wave packet.

The initial conditions for the scalar field are "trivial,"

$$
\phi(t=0, \mathbf{x})=\eta, \quad\left[\partial_{t} \phi(t, \mathbf{x})\right]_{t=0}=0 .
$$

The free parameters in the initial conditions are $z_{0}, a, w$, $\omega$, and $\omega^{\prime}$. For our simulations, we will rescale these parameters as follows:

$$
\begin{aligned}
z_{0} & =\frac{\bar{z}_{0}}{\eta}, & & a=\frac{\bar{a}}{\eta}, \\
\omega & =\bar{\omega} \eta, & \omega^{\prime} & =\bar{\omega}^{\prime} \eta .
\end{aligned}
$$

The dimensionless parameters $\bar{z}_{0}, \bar{a}, \bar{w}, \bar{\omega}$, and $\bar{\omega}^{\prime}$ above are varied in our code. In addition, the Abelian Higgs model has the parameters $e, \lambda$, and $\eta$. However, by field and coordinate rescalings, there is only one model parameter given by the ratio of scalar and vector masses, $\beta=m_{S}^{2} / m_{V}^{2}=2 \lambda / e^{2}$. 


\section{COMPUTATIONAL TECHNIQUES}

Following the numerical relativity based approach developed in [1], we introduce a new dynamical variable $\Gamma=\partial_{i} A_{i}$. Then the field variables are $\phi, A_{i}$, and $\Gamma$, altogether 6 functions. The equations of motion for these variables are

$$
\begin{aligned}
& \partial_{t}^{2} \phi_{a}= \nabla^{2} \phi_{a}-e^{2} A_{i} A_{i} \phi_{a}-2 e \epsilon_{a b} \partial_{i} \phi_{b} A_{i}-e \epsilon_{a b} \phi_{b} \Gamma \\
&- \lambda\left(\phi_{b} \phi_{b}-\eta^{2}\right) \phi_{a} \\
& \partial_{t} F_{0 i}= \nabla^{2} A_{i}-\partial_{i} \Gamma+e\left(\epsilon_{a b} \phi_{a} \partial_{i} \phi_{b}+e A_{i} \phi_{a} \phi_{a}\right) \\
& \partial_{t} \Gamma=\partial_{i} F_{0 i}-g_{p}^{2}\left[\partial_{i} F_{0 i}+e \epsilon_{a b} \phi_{a} \partial_{t} \phi_{b}\right]
\end{aligned}
$$

where $a=1,2, \epsilon_{a b}$ is the Levi-Civita tensor with $\epsilon_{12}=1$, $F_{0 i}=\partial_{t} A_{i}$ in the temporal gauge, and $g_{p}^{2}$ is a new parameter introduced for numerical stability. The idea is that the square bracket in Eq. (21) vanishes in the continuum because of the Gauss constraints $\nabla \cdot \mathbf{E}=\rho$ where $\rho$ is the charge density. However, the square bracket may not vanish upon discretization. By writing the equations in the above form with the auxiliary function $\Gamma$, we obtain improved numerical stability as is also seen in numerical relativity [2]. The value of the parameter $g_{p}^{2}$ is chosen by numerical experimentation; we have set $g_{p}^{2}=$ 0.75 in our simulations. The initial conditions for the auxiliary function $\Gamma$ follow from the choice of initial conditions for the gauge field,

$$
\Gamma(t=0, \mathbf{x})=0 .
$$

For our analysis, we discretized these equations on a $256^{3}$ lattice with lattice spacing $\Delta x=0.05$ and time step size $\Delta t=\Delta x / 4$. The difference equations were solved using the explicit Crank-Nicholson method with two iterations. To reduce computation times, we parallelized our numerical code. As a check of our evolution code, we find that the total energy inside the box is conserved to within $1 \%$ during the entire evolution period.

In addition to the evolution of equations, we developed a tracking code which detects strings and calculates the number of loops that are present in the simulation domain at any given time. The program calculates the phase winding as defined in Eq. (5) on every plaquette of the lattice. A nonzero winding on a plaquette implies that a string passes through the plaquette and enters/exits the corresponding cells. The program then connects the strings and records the properties of the loops.

The string tracking algorithm is the same as used in earlier work [3,4] but with one subtlety. In calculating the winding as in Eq. (5), we have to find the discretized value of $d \theta$ along the links of the lattice. Generally one uses the "geodesic rule" and the phase difference between lattice sites $i$ and $i+1$ is

$$
d \theta \rightarrow \Delta \theta \equiv \theta_{i+1}-\theta_{i}+2 \pi k
$$

where $k=0, \pm 1$ is chosen to minimize $|\Delta \theta|$. However, this rule ignores the case when $|\Delta \theta|=\pi$. The justification in earlier works has been that this possibility is of zero measure. In our case, however, this situation arises quite frequently. The reason can be seen from the equations of motion and the initial conditions. We start out with $\phi_{1}=\eta$ and $\phi_{2}=0$, i.e., $\theta=0$ throughout the lattice. The equations of motion are such that they tend to preserve $\phi_{2}=0$, and all the nontrivial dynamics is in the $\phi_{1}$ variable, at least at early times. Now $\phi_{1}$ can become negative. When $\phi_{1}$ differs in sign at neighboring lattice sites, this gives a phase difference of exactly $\pm \pi$ and the geodesic rule is ambiguous. In evaluating the winding number, we choose $+\pi$ or $-\pi$ with equal probability.

\section{RESULTS}

As we have discussed above, the problem contains 1 model parameter, namely, $\beta$, and 5 initial condition parameters. We will fix some of these parameters and scan over a range of a few parameters. We set

$$
\overline{z_{0}}=1.8, \quad \bar{w}=0.6, \quad \bar{\omega}^{\prime}=\bar{\omega} .
$$

We have explored

$$
\beta \in[0.08,8.0], \quad \bar{a} \in[0.6,7.0], \quad \bar{\omega} \in[0.2,8.0] .
$$

We did not see any qualitative changes as we varied $\beta$ (see below) and so for most of our runs we set $\beta=1$, equivalently $e=0.5, \lambda=0.125$. We also chose $\eta=1$ and this sets the length scale in the simulation.

From our initial runs, we found that energy is condensed into strings even from a single wave packet, i.e., without scattering two gauge wave packets. We will call this "prompt string production" and it is reminiscent of the discovery in [5] that strings may be formed due to purely gauge field fluctuations during a phase transition. In the next subsection, we will explore prompt string production and find that there are regions of parameter space where prompt production does not occur. We will then move on to explore this region of parameter space and find a subregion where strings are produced when wave packets collide.

\section{A. Prompt string production}

For the single pulse case, after fixing the parameters of the theory, we managed to analytically find expressions for the total energy in the simulation domain for the wave packet profiles shown in the previous section. It is as follows:

$$
E_{\text {Single }}=\frac{\bar{a}^{2} \pi^{\frac{3}{2}} \eta\left(9+10 \bar{w}^{2} \bar{\omega}^{2}+4 \bar{w}^{4} \bar{\omega}^{4}+2 e^{2}\left(\bar{w}^{2}+\bar{w}^{4} \bar{\omega}^{2}\right)\right)}{8 \bar{w}^{3}} .
$$


$\odot$

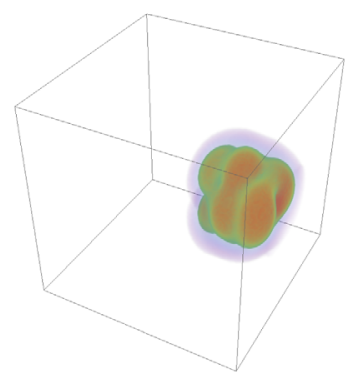

100

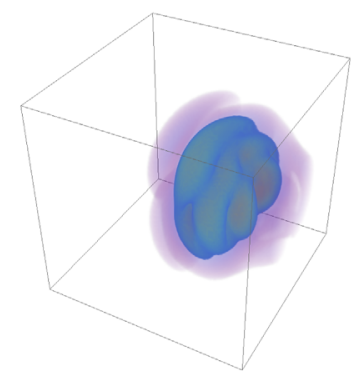

200

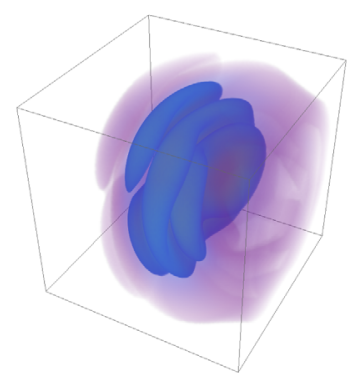

50

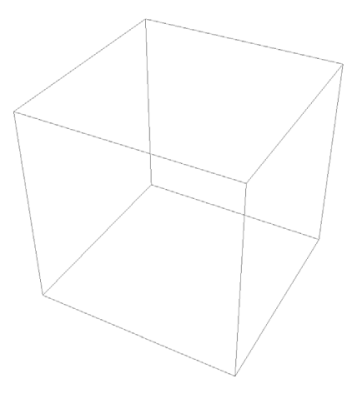

$15 \odot$
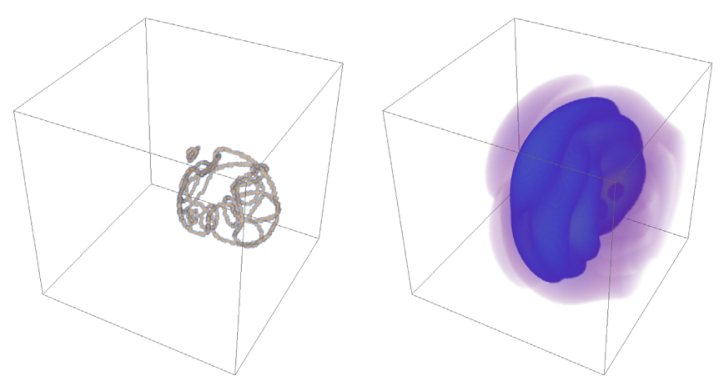

250

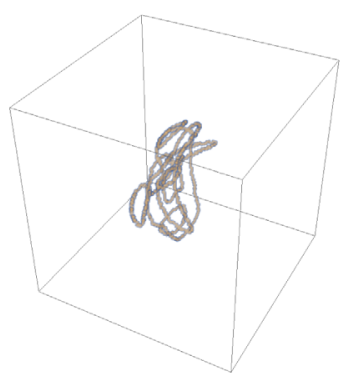

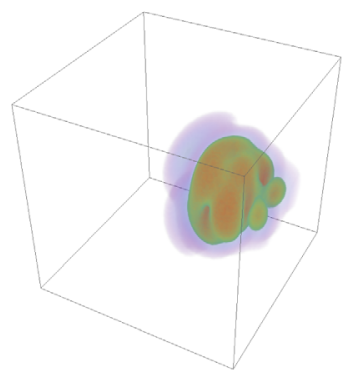
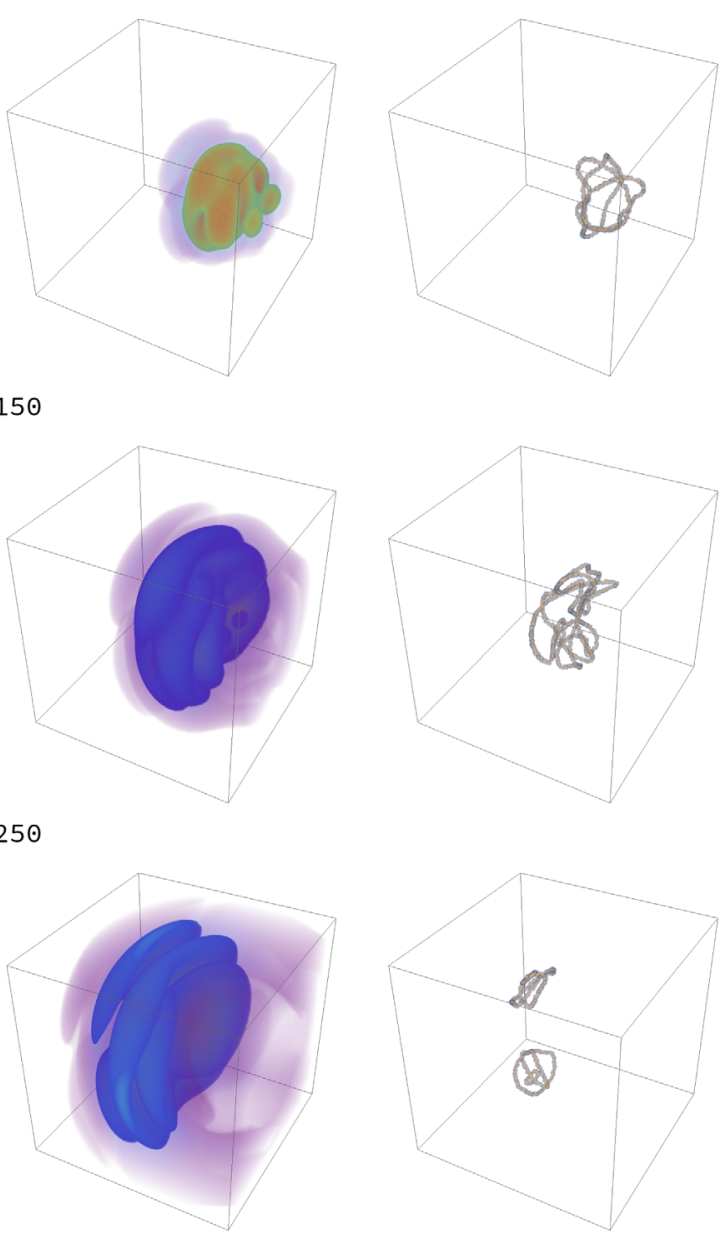

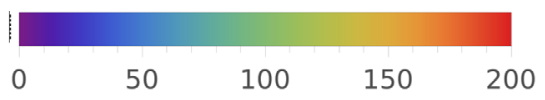

FIG. 1. Total energy density (boxes on the left) and winding (boxes on the right) at different time steps for the case of one pulse for $\bar{a}=6.215, \bar{\omega}=2.0, \lambda=0.125$, and $E_{\text {Single }}=4000$. The $116^{3}$ boxes shown here are smaller than the full lattice $\left(256^{3}\right)$.

With this expression, we can trade one of the parameters for the total energy.

In Fig. 1 we show the prompt production of strings at various times during the evolution. In the first frame, there is energy density of the wave packet but no strings. Some time steps later, the scalar field has adjusted to the gauge wave packet and strings, as detected by topological winding, are produced. As the system evolves further, the dense network of strings chops itself up and decays.

We have examined prompt production for several different values of the model parameter $\lambda$ (equivalently $\beta$ since we fix $e=0.5$ ). Figure 2 shows how the length in strings - evaluated by counting the plaquettes that contain nontrivial topological winding - changes with time. The figure shows that the outcome is not very sensitive to the value of $\lambda$ and hence we set $\lambda=0.125(\beta=1)$ in the runs described below.
In contrast, as seen in Fig. 3, the prompt production of strings depends sensitively on the parameter $\bar{\omega}$. The general trend is that less length is produced for larger $\bar{\omega}$ but the strings that are produced survive for a longer time. This can happen if larger $\bar{\omega}$ leads to larger loops or to loops with higher angular momentum.

In Fig. 4 we plot the energy density integrated over $\bar{x}$ and $\bar{y}$ coordinates, as a function of $\bar{z}$. Prompt string production occurs at the initial location of the wave packet $\left(\bar{z}_{0}=1.8\right.$ or 36 lattice spacings away from the center of the lattice). Then, the string cluster moves towards the left and also decays.

We have calculated the length of strings at any given time by counting the number of plaquettes with nontrivial winding. We can also estimate the energy in the string network by adding up the field energies in all the cells within $m_{S}^{-1}$ or $m_{V}^{-1}$ (whichever is larger) of the string 


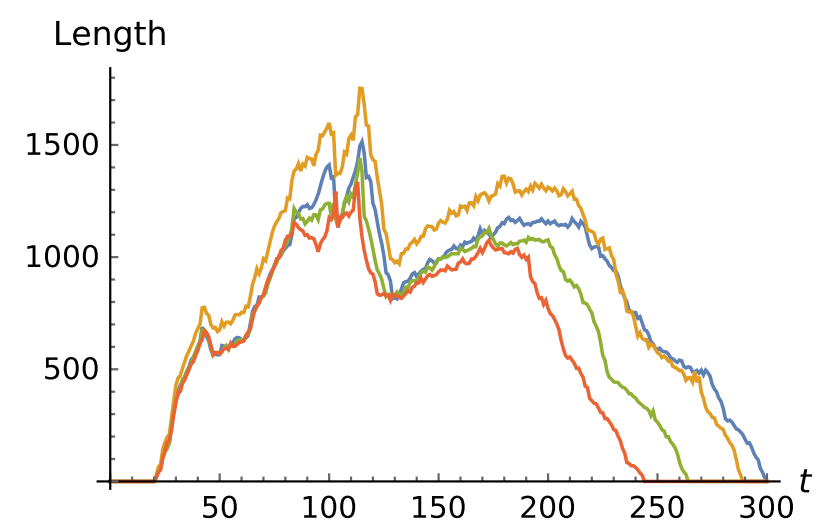

FIG. 2. Total length of strings (in units of number of lattice points) as a function of time(-steps) for $\lambda=0.01$ (blue), $\lambda=$ 0.125 (orange), $\lambda=0.50$ (green), and $\lambda=1.0$ (red). All the other kinematic parameters are kept fixed with $\bar{a}=6.215$ and $\bar{\omega}=2.0$.

network. However, the plot in Fig. 5 of the energy versus time shows reasonable correspondence with the length versus time plot in Fig. 3 for $\bar{\omega}=2.0$, indicating that the strings do not have significant kinetic energy at formation.

As expected, greater initial energy produces more strings. However, our analysis indicates some subtleties in the process of string production. From Eq. (26), it can be seen that, for fixed energy, the amplitude becomes smaller as we increase the frequency and vice-versa. (The wave packet width $\bar{w}$ is fixed in all our runs.) After experimenting with different values of amplitude and frequency at fixed energy, we noticed that there is a minimum/critical amplitude below which we do not produce any strings (as seen in Fig. 6). The parameter space under the critical curve, for which strings are not produced, gets smaller as the energy increases. In the opposite limit of small $\bar{\omega}$ (large amplitude), we see that the total length of strings is far greater (also seen in Fig. 3). The physical origin of this behavior is not clear.

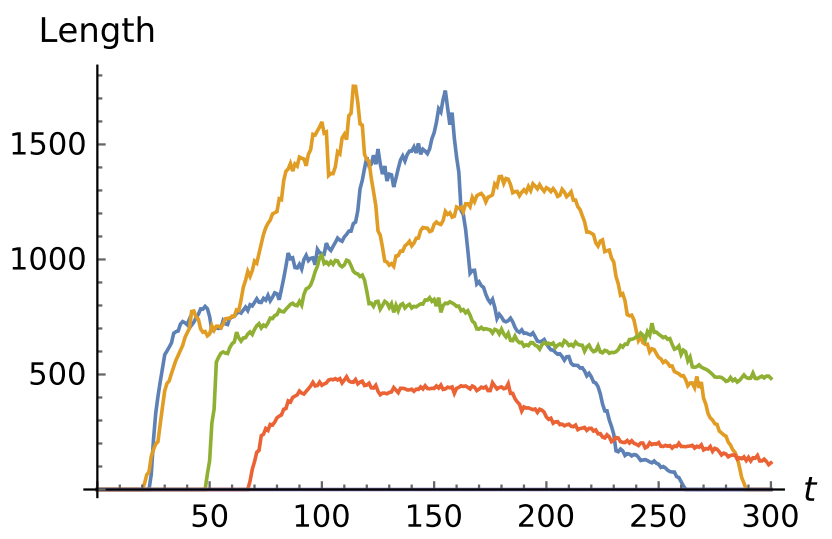

FIG. 3. Total length of strings (in units of number of lattice points) as a function of time(-steps) for $\bar{\omega}=0.1$ (blue), $\bar{\omega}=2.0$ (orange), $\bar{\omega}=4.0$ (green), $\bar{\omega}=6.0$ (red), $\bar{\omega}=8.0$ (no strings), and $\lambda=0.125$. Total energy for all the runs is kept fixed at, $E_{\text {Single }}=4000$, by adjusting $\bar{a}$ suitably according to Eq. (26).

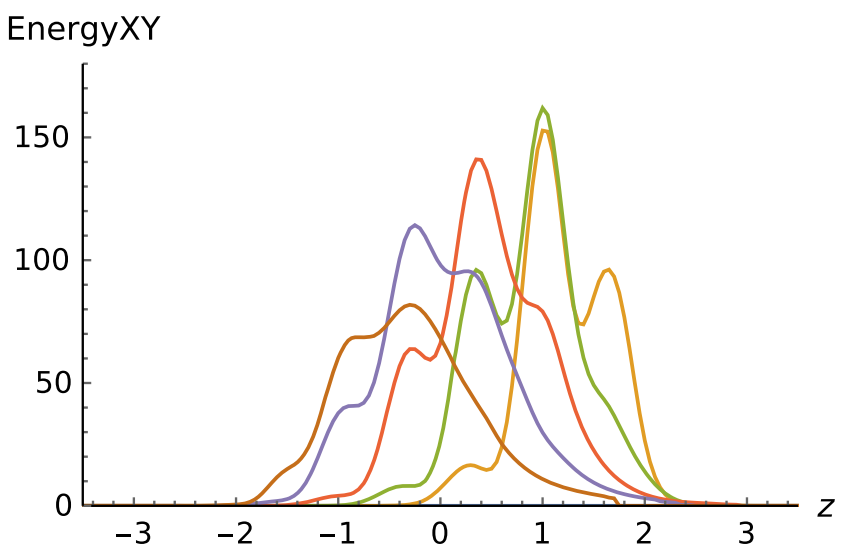

FIG. 4. Energy in the strings in the $x y$ planes as a function of $z$ at time steps $t=0$ (no strings), $t=50$ (orange, right-most curve), $t=100$ (green), $t=150$ (red), $t=200$ (blue), and $t=250$ (brown, left-most curve), during the simulation for $\bar{a}=6.215$, $\bar{\omega}=2.0, \lambda=0.125$, and $E_{\text {Single }}=4000$. Following prompt string production, the string network moves to the left and decays.

One expectation is that more strings are produced if there is higher energy density (for the same total energy). However, upon plotting the maximum energy density in our simulation box over the duration of the run with respect to

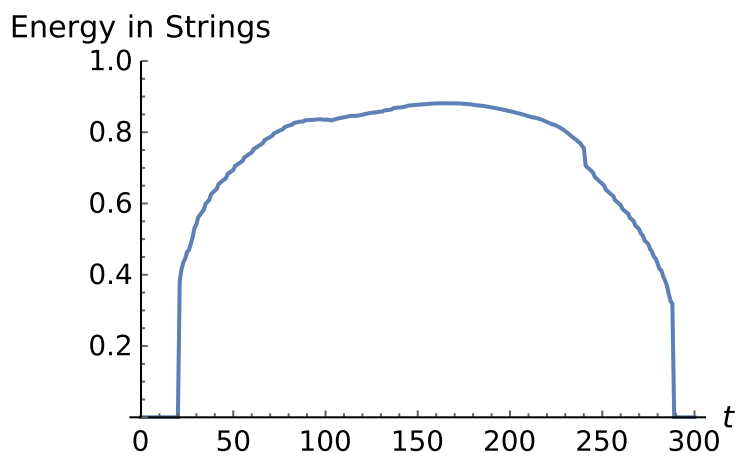

FIG. 5. Energy in strings as a fraction of total energy versus time(-steps) for $\bar{a}=6.215, \bar{\omega}=2.0, \lambda=0.125$, and $E_{\text {Single }}=4000$.

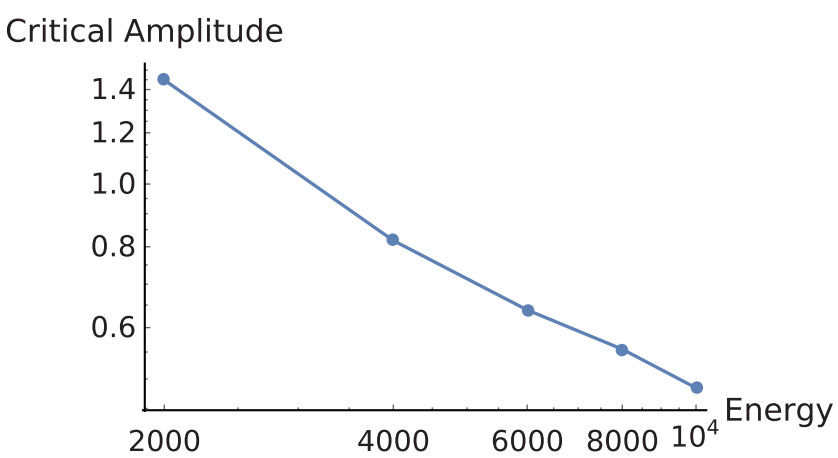

FIG. 6. Critical amplitude $\bar{a}$ as we change total input energy, $E_{\text {Single }}$, for the single pulse case with $\lambda=0.125$. Strings are only produced above the curve. 
Maximum Energy Density

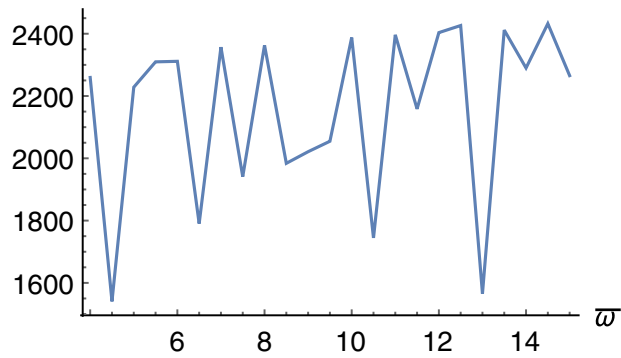

FIG. 7. Maximum energy density as a function of frequency $\bar{\omega}$ in the initial wave packets for $\lambda=0.125$ and total energy of 4000 in the box. The critical frequency above which no strings are produced here is 7.5 (corresponding to $\bar{a}=0.817$ ).

$\odot$

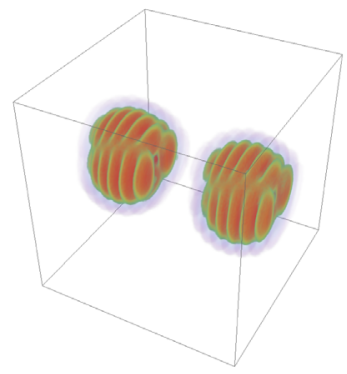

153

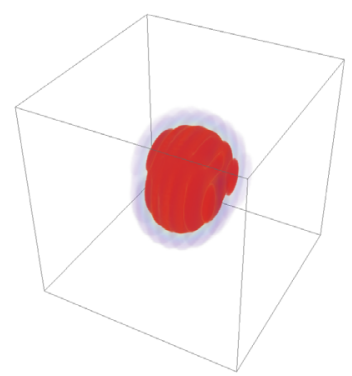

193

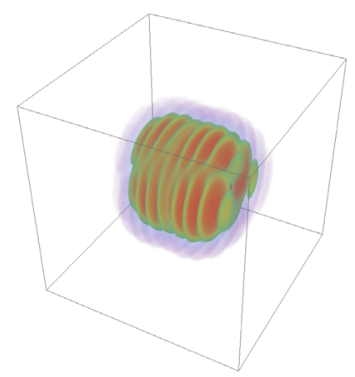

frequency (for fixed total energy equal to 4000), we find noisy behavior with an overall increasing trend (see Fig. 7). This is counterintuitive, since no strings are produced above the critical value of $\bar{\omega}=7.5$ (corresponding to the critical amplitude $\bar{a}=0.817$ as seen in Fig. 6). This indicates that energy density alone may not determine string production and is reminiscent of the chaotic behavior seen in kink production in $1+1$ dimensions [6,7].

\section{B. Wave packet collisions}

We now consider the case when two wave packets collide. The parameters are chosen so that there is no prompt string production. However, strings are produced when the wave packets collide. So now we have two wave packets in the initial conditions that are headed towards a collision. The initial energy is

133
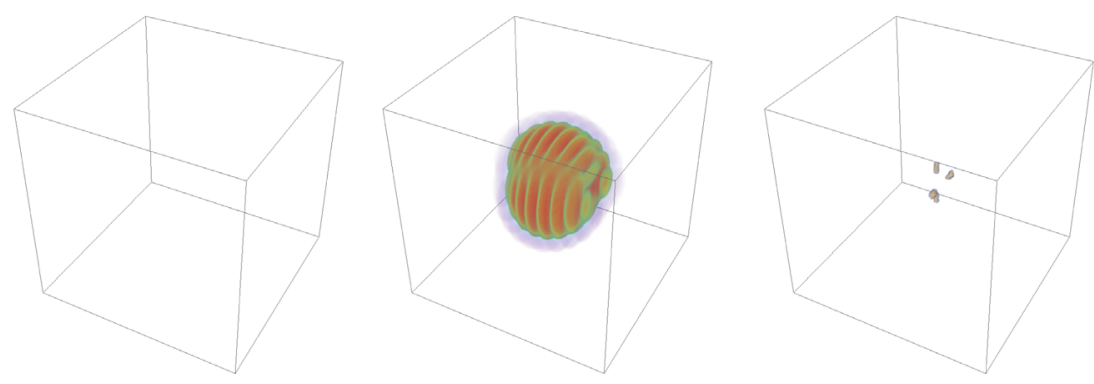

173
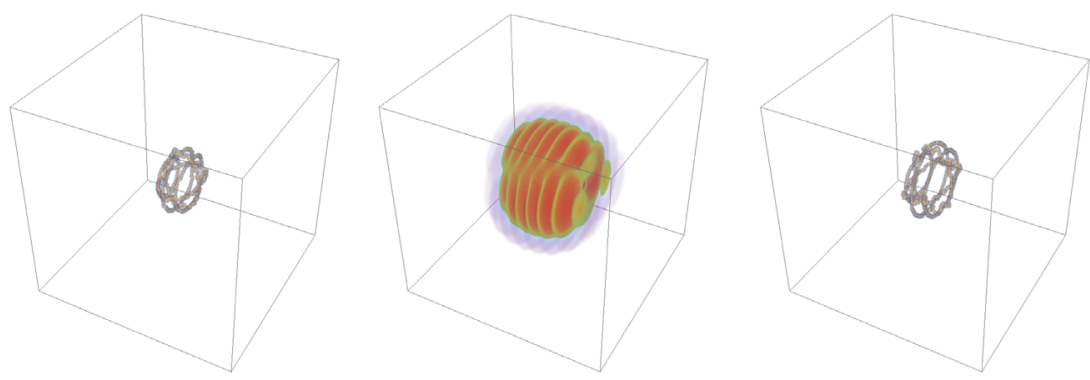

213
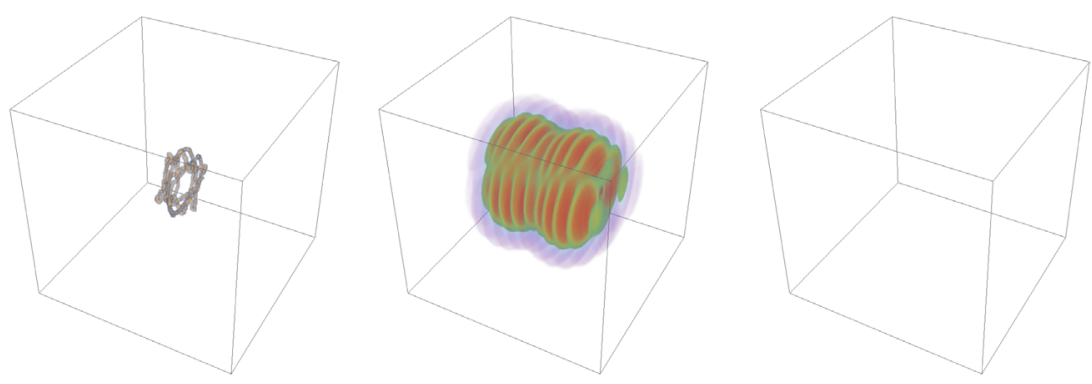

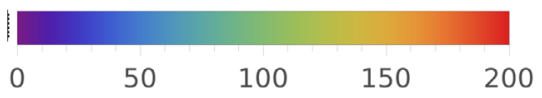

FIG. 8. Total energy density (boxes on the left) and winding (boxes on the right) at different time steps for the case of two collinear pulses for $\bar{a}=0.578, \bar{\omega}=9.0, \lambda=0.125$, and $E_{\text {Double }} \approx 8000$. The strings are first produced at time step 133 in our simulation, and therefore we have not shown plots for intermediate time steps. The $116^{3}$ boxes shown here are smaller than the full lattice $\left(256^{3}\right)$. 


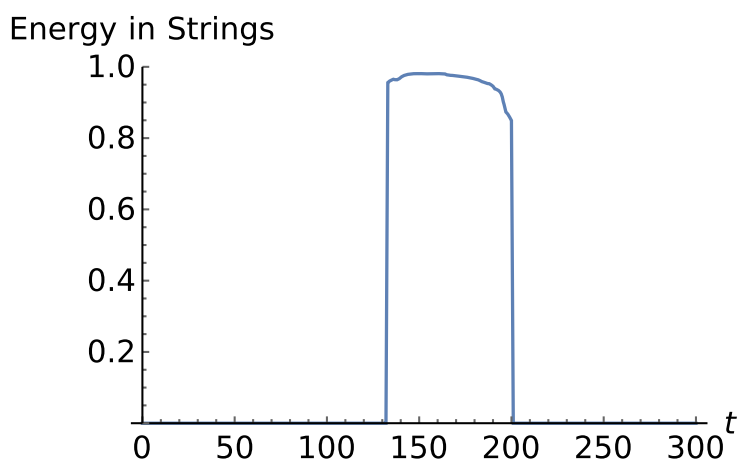

FIG. 9. Energy in strings as a fraction of total energy versus time for $\bar{a}=0.578, \bar{\omega}=9.0, \lambda=0.125$, and $E_{\text {Double }} \approx 8000$.

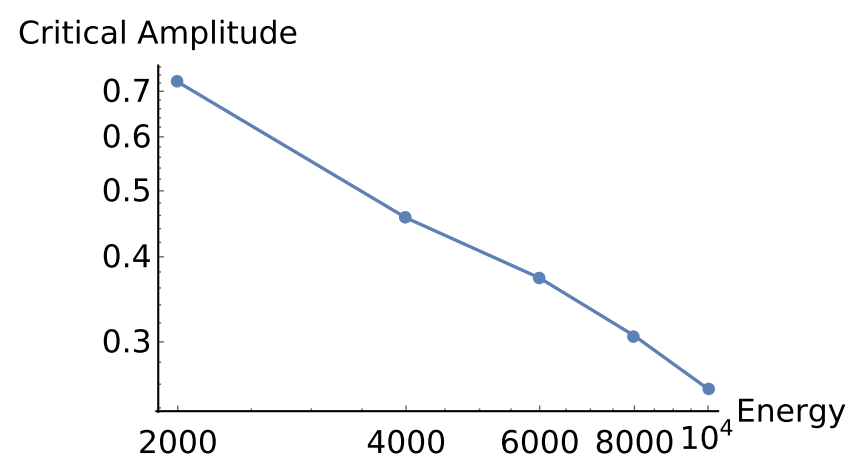

FIG. 10. Critical amplitude $\bar{a}$ as we change total input energy, $E_{\text {Double }}$, for the case of colliding wave packets for $\lambda=0.125$. Strings are only produced above the curve.

$$
\begin{aligned}
E_{\text {Double }}= & 2 E_{\text {Single }}+\frac{\pi^{3 / 2} \bar{a}^{2} \eta}{4 \bar{w}^{7}} e^{-\bar{z}_{0}^{2} / \bar{w}^{2}}\left[-18 \bar{w}^{2} \bar{z}_{0}^{2}+4 \bar{z}_{0}^{4}\right. \\
& +2 \bar{w}^{8} \bar{\omega}^{2}\left(e^{2}+2 \bar{\omega}^{2}\right)+2 \bar{w}^{6}\left(e^{2}+5 \bar{\omega}^{2}\right) \\
& +\bar{w}^{4}\left(9-2 e^{2} \bar{z}_{0}^{2}-8 \bar{z}_{0}^{2} \bar{\omega}^{2}\right) \cos \left(2 \bar{z}_{0} \bar{\omega}\right) \\
& \left.-8 \bar{w}^{2} \bar{z}_{0} \omega\left(2 \bar{w}^{2}-\bar{z}_{0}^{2}+\bar{w}^{4} \bar{\omega}^{2}\right) \sin \left(2 \bar{z}_{0} \bar{\omega}\right)\right] .
\end{aligned}
$$

We again use Eq. (26) for fixing kinematic parameters. For the simulation, we chose $\bar{a}=0.578$ and $\bar{\omega}=9.0$ for the individual wave packets. With this choice prompt production of strings does not occur, that is, the parameters lie below the critical curve for the single pulse case shown in Fig. 6.

Figure 8 shows the evolution of the wave packets and string formation after collision. Very few short-lived strings are produced even though the total input energy is much higher $(\approx 8000)$ compared to the single pulse run presented in the previous subsection. The fractional energy in strings as a function of time is shown in Fig. 9. By scanning over different amplitudes, $\bar{a}$, for the same total energy, we find the critical curve for string formation when wave packets collide. The critical curve is plotted in Fig. 10.

\section{CONCLUSIONS}

We have explored the formation of U(1) gauge strings due to wave packets of gauge fields in two settings: (i) the prompt formation of strings from gauge fields, and (ii) the formation of strings when gauge wave packets collide. We have restricted our attention to a class of wave packets with certain parameters, and found critical curves in parameter space that demarcate string formation regions. These critical curves show that it is easier to produce strings with higher energy wave packets (see Figs. 6 and 10). However, we have not found a general pattern beyond this simple conclusion. The reason may lie in the chaotic behavior observed in previous studies of kink production in $1+1$ dimensions [6,7].

It is also interesting to contrast string production with magnetic monopole production. Unlike the case of magnetic monopoles, the string loops that are formed are short lived as they collapse and produce radiation. The loops may live longer if we could find initial conditions that provide them with greater angular momentum but these too will not live indefinitely. On the other hand, once a magnetic monopole and antimonopole pair are produced with sufficient velocity, they will move apart and survive indefinitely. Furthermore, magnetic monopoles are localized objects and so the colliding wave packets need not be very extended. For strings, the wave packets have to extend over a region that is the size of the string loop that is to be produced, and only relatively small loops can be produced. In these respects it appears that magnetic monopoles are easier to produce than strings.

The flip side is that we know systems that contain gauge strings while the existence of magnetic monopoles is still speculative. Gauge strings are known to exist in superconductors and, in that setting, our gauge field wave packets correspond to photon wave packets. This suggests that by shining light on superconductors we could produce strings within the superconductor. However, a realistic superconductor is described by a different set of equations that take into account the dependence of the model parameters on the temperature [8]. It will be interesting to adapt our analysis to study string production in superconductors.

\section{ACKNOWLEDGMENTS}

We are grateful to Onur Erten for remarks. The computations were done on the A2C2 Saguaro and Agave clusters at Arizona State University. This work is supported by the U.S. Department of Energy, Office of High Energy Physics, under Award No. DE-SC0018330 at Arizona State University. 
[1] T. Vachaspati, Phys. Rev. Lett. 117, 181601 (2016).

[2] T. W. Baumgarte and S. L. Shapiro, Numerical Relativity: Solving Einstein's Equations on the Computer (Cambridge University Press, New York, 2010).

[3] T. Vachaspati and A. Vilenkin, Phys. Rev. D 30, 2036 (1984).

[4] L. Pogosian and T. Vachaspati, Phys. Lett. B 423, 45 (1998).
[5] M. Hindmarsh and A. Rajantie, Phys. Rev. Lett. 85, 4660 (2000).

[6] S. Dutta, D. A. Steer, and T. Vachaspati, Phys. Rev. Lett. 101, 121601 (2008).

[7] T. Romanczukiewicz and Ya. Shnir, Phys. Rev. Lett. 105, 081601 (2010).

[8] D. M. Kennes and A. J. Millis, Phys. Rev. B 96, 064507 (2017). 